\title{
Suppression of Cross-Gain Modulation in SOA Using RZ-DPSK Modulation Format
}

\author{
Pak S. Cho and Jacob B. Khurgin
}

\begin{abstract}
Conventional ON-OFF keyed modulation format is susceptible to cross-gain modulation in saturated semiconductor optical amplifier (SOA) that leads to crosstalk penalty for dense wavelength-division-multiplexing (DWDM) input signals. Differential phase-shift keying of optical pulses have virtually no pulse-pattern effect and are robust to cross-gain modulation. We report experimental results that confirm reduction of crosstalk penalty in SOA with differential phase-shift-keyed DWDM input signals in comparison with ON-OFF keyed signals operating at 12.5 Gb/s with $25-\mathrm{GHz}$ channel spacing.
\end{abstract}

Index Terms-Crosstalk, differential phase-shift keying (DPSK), phase-shift keying, semiconductor optical amplifiers (SOAs), wavelength-division multiplexing.

\section{INTRODUCTION}

$\mathbf{T}$ HE ADVANCE of optical amplifiers makes high-capacity dense wavelength-division-multiplexing (DWDM) transmission practical and economically feasible for terrestrial and undersea fiber links. Optical amplifiers such as erbium-doped fiber amplifiers (EDFAs) are widely used because of its low noise performance and high output power capabilities. Semiconductor optical amplifiers (SOAs) have the advantage of broad gain spectrum $(>60 \mathrm{~nm})$ and they are compact and integrable. However, SOA suffers cross-gain modulation (XGM) that results in crosstalk penalty for DWDM input signals [1]. This is due to its relatively low saturation energy and a gain recovery time comparable with the bit period. Various techniques were reported to improve SOAs saturation characteristics [2], [3]. Conventional ON-OFF keyed (OOK) signals are particularly susceptible to XGM in SOAs. In fact, XGM is so efficient for OOK signals that SOAs are useful for all-optical wavelength conversion [4], [5]. Constant-intensity modulation format such as phase-shift keying is in principle immune to XGM-induced crosstalk in SOAs [6]. Recent reported results on return-to-zero differential phase-shift-keyed (RZ-DPSK) modulation format had shown that it is more tolerant to fiber nonlinearities due in part to its continuous pulse sequence with no missing pulses [7]-[9]. DPSK had been used in combination with cascaded SOA in a single-channel experiment and the reduction of pattern effect had been observed [6]. Therefore, RZ-DPSK signal with virtually no data-induced pulse pattern should be more tolerant to XGM in SOA than OOK signal for

Manuscript received July 30, 2002; revised September 12, 2002.

P. S. Cho is with CeLight, Inc., Silver Spring, MD 20904 USA.

J. B. Khurgin is with the Department of Electrical and Computer Engineering,

John Hopkins University, Baltimore, MD 21218 USA.

Digital Object Identifier 10.1109/LPT.2002.805795

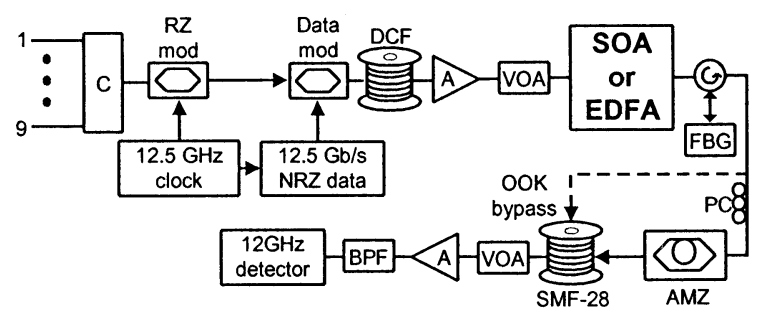

Fig. 1. Experimental setup for crosstalk measurement. C: coupler; DCF: bit decorrelation fiber; A: EDFA; PC: polarization controller. AMZ: asymmetric Mach-Zehnder demodulator; BPF: bandpass filter.

DWDM system. In this letter, we report experimental results that confirm the reduction of SOA crosstalk penalty using RZ-DPSK format compared with RZ-OOK format using nine $25-\mathrm{GHz}$-spaced channels operating at $12.5 \mathrm{~Gb} / \mathrm{s}$. This is, to the best of our knowledge, the first reported result on SOA with RZ-DPSK input DWDM signals with 25-GHz channel spacing. Our results proved that RZ-DPSK signals are more tolerant to XGM in SOA than OOK signals and, as a result, the design of SOA can be relaxed for RZ-DPSK signals.

\section{EXPERIMENTAL SETUP AND RESULTS}

Fig. 1 shows the experimental setup. Nine distributed feedback (DFB) lasers were combined and launched into a lithium niobate (LN) Mach-Zehnder modulator (MZM) driven by a $12.5-\mathrm{GHz}$ sinusoidal wave to produce a $12.5-\mathrm{GHz}$ optical pulse train with a duty cycle of $60 \%$. The pulses were encoded with DPSK or OOK format using a push-pull LN MZM biased at null or quadrature driven by a $12.5-\mathrm{Gb} / \mathrm{s}$ nonreturn-to-zero (NRZ) data stream with a drive voltage of $V_{\pi}$ or $2 V_{\pi}$. The NRZ data was a pseudorandom binary sequence (PRBS) with a word length of $2^{31}-1$. A coil of fiber with approximately $-340 \mathrm{ps} / \mathrm{nm}$ of dispersion was used to decorrelate the nine 25-GHz-spaced channels. The nine DWDM channels were then amplified using an EDFA followed by a variable optical attenuator (VOA) to control the input power to the SOA. The SOA was biased at $200 \mathrm{~mA}$ at $20^{\circ} \mathrm{C}$. The optical signal-to-noise ratio (OSNR) of the input signal to the SOA was better than $35 \mathrm{~dB}$ $(0.1 \mathrm{~nm})$ for both OOK and DPSK cases. The InGaAsP-based fiber-pigtailed SOA is a commercial device with a small-signal gain (fiber-to-fiber) of $22 \mathrm{~dB}$. The saturation output power was $+5 \mathrm{dBm}$. The input power for saturation was about $-14 \mathrm{dBm}$. The tensile strained SOA with angled facet has a low polarization differential gain of $0.2 \mathrm{~dB}$ at $1550 \mathrm{~nm}$. The noise figure (NF) of the SOA was at least $6.5 \mathrm{~dB}$ at $-25 \mathrm{dBm}$ input power. 


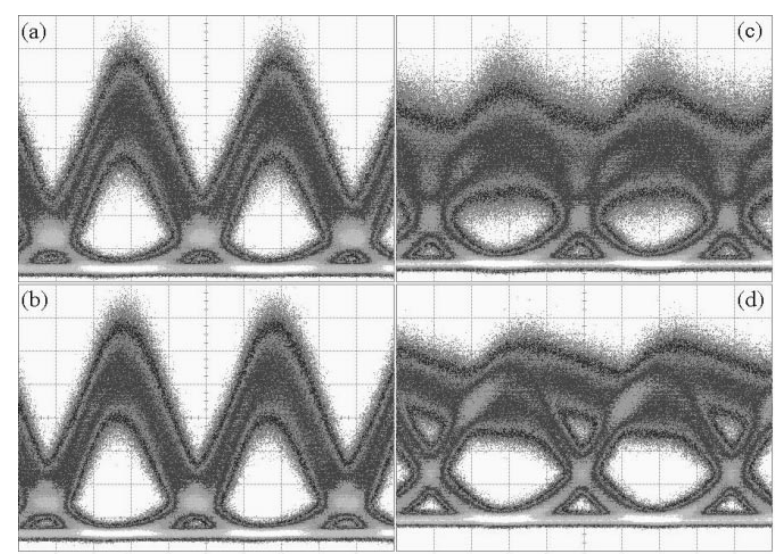

Fig. 2. Received eye diagrams of the center channel for DPSK input to (a) SOA or (b) EDFA, and for OOK input to (c) SOA or (d) EDFA. The composite input power to the SOA was $-17 \mathrm{dBm}$ for both DPSK and OOK signals. PRBS length: $2^{31}-1$. Horizontal scale: $20 \mathrm{ps} /$ div.

The amplified output of the SOA was directed to a fiber Bragg grating filter (FBG) through a circulator to select the center channel at $1545.32 \mathrm{~nm}$. The FBG filter was $18.2 \mathrm{GHz}$ wide and no significant adjacent channel crosstalk was observed. $20 \mathrm{~km}$ of SMF-28 fiber $(+340 \mathrm{ps} / \mathrm{nm})$ was used to compensate the dispersion of the decorrelating fiber. The SMF-28 fiber was placed after the FBG filter to avoid fiber nonlinearities such as four-wave mixing. The signal was then directed to a receiver consists of an optical pre-amp (EDFA), a 1.3-nm optical bandpass filter, and a 12-GHz photoreceiver. For DPSK signal, an asymmetric Mach-Zehnder (AMZ) device was inserted after the FBG filter to demodulate the DPSK signal. The polarization sensitive AMZ demodulator has a differential delay of $80 \mathrm{ps}$ and a differential phase shift controlled by a thermo-electric cooler. Due to imperfections of the FBG filter spectral response dispersion penalty caused by inter-symbol interference was observed for OOK signal. For DPSK signal, the degradation of the FBG was partly compensated for by the AMZ demodulator [9].

In order to distinguish the XGM-induced crosstalk effect of the SOA from its amplified spontaneous emission (ASE) noise degradation, measurements were repeated with the SOA replaced with an EDFA. The OSNR of the EDFA output was adjusted to be the same as for the case of the SOA by controlling the input power to the EDFA. The EDFA has a small-signal gain of more than $37 \mathrm{~dB}$ and an NF of $3.6 \mathrm{~dB}$ at $-25 \mathrm{dBm}$ input. Fig. 2 shows eye diagrams of the center channel detected at the receiver for the case of DPSK and OOK signals amplified by SOA or EDFA. The composite input power to the SOA was $-17 \mathrm{dBm}$ for both DPSK and OOK signals. The degradation due to the SOA for DPSK signal was minimal compared with the case of EDFA as can be seen in Fig. 2(a) and (b). For OOK signals, XGM degradation in SOA as a result of pulse-pattern effect can be clearly seen in Fig. 2(c) and (d). Note again that due to the FBG filter and the AMZ demodulator response the DPSK and OOK signals are different even with EDFA as can be seen in Fig. 2(b) and (d). Fig. 3 shows snap shots of bit patterns of the received DPSK (top) and OOK (bottom) signals amplified by SOA (solid lines) or EDFA (dotted lines). The top, middle, and bottom

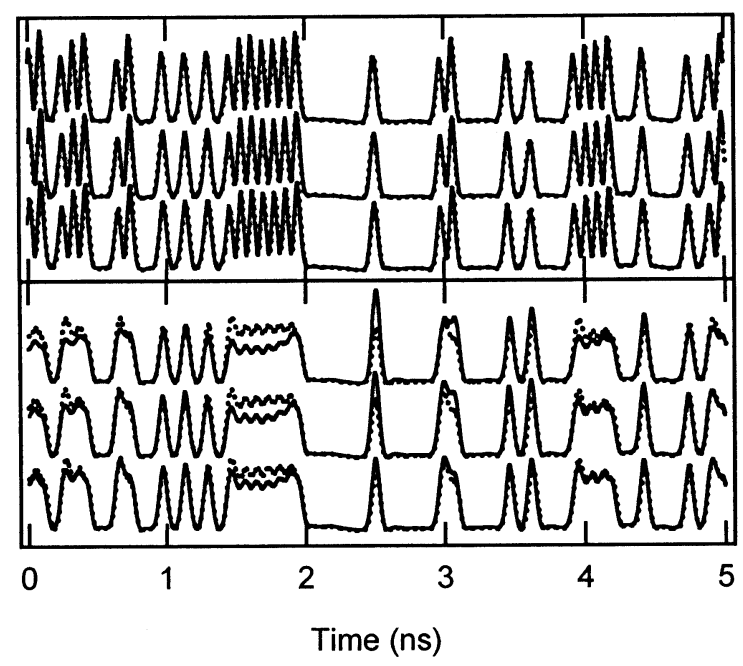

Fig. 3. Time-averaged bit pattern of the detected DPSK (top graph) and OOK (bottom graph) signals at the receiver. The top, middle, and bottom traces of each graph correspond to composite input powers of $-14,-17$, and $-20 \mathrm{dBm}$ to the SOA. The solid and dotted lines depict the cases for SOA and EDFA, respectively. PRBS length: $2^{7}-1$.

traces correspond to composite input powers of $-14,-17$, and $-20 \mathrm{dBm}$ into the SOA. The PRBS length was $2^{7}-1$. The displayed bit patterns were time averaged to minimize the ASE noise so it does not mask the XGM-induced patterning effect. Severe pulse-patterning degradation of the OOK signal as a result of XGM can be clearly seen as the input power to the SOA increases. The severe pulse amplitude variation is a clear manifestation of XGM. DPSK signal, on the other hand, suffers virtually no XGM degradation for all three input powers and is almost indistinguishable from the case of EDFA as can be seen in Fig. 3. Note that no pulse-pattern effect due to XGM was observed for DPSK signal when the AMZ demodulator was removed. Therefore, the AMZ demodulator does not take part in the suppression of XGM.

Bit error rate (BER) measurements of the received center channel were performed for both DPSK and OOK signals amplified by SOA or EDFA as shown in Fig. 4 for input powers of $-14,-17$, and $-20 \mathrm{dBm}$ into the SOA. For the case of EDFA, the input power was adjusted to produce the same output OSNR as for the case of SOA. The input powers to the EDFA were $-21,-23$, and $-24 \mathrm{dBm}$. From the BER curves, DPSK signal clearly outperforms OOK signal. The change in the slope of the BER curve at higher received power is due to the limitation of our receiver (limited gain and output power of the optical pre-amp) at low OSNR input. The OSNR of the SOA output was less than $22 \mathrm{~dB}(0.1 \mathrm{~nm})$ at $-17-\mathrm{dBm}$ input to the SOA as a result of its relatively high NF ( $>6.5 \mathrm{~dB})$. No change in the slope of the BER curve was observed with higher OSNR input signal. The optimal input power to the SOA was about $-20 \mathrm{dBm}$ for OOK signal. Higher input power causes more XGM degradation and lower input power leads to more ASE noise penalty. For DPSK signal, the optimal input power was about $-17 \mathrm{dBm}$. The 3-dB higher value of the optimal power for DPSK could be due to its lower pulse-peak power (no missing pulses) for the same average power as the OOK signal. Even though no pulse-pattern effect was observed for input powers up to $-10 \mathrm{dBm}$ for 

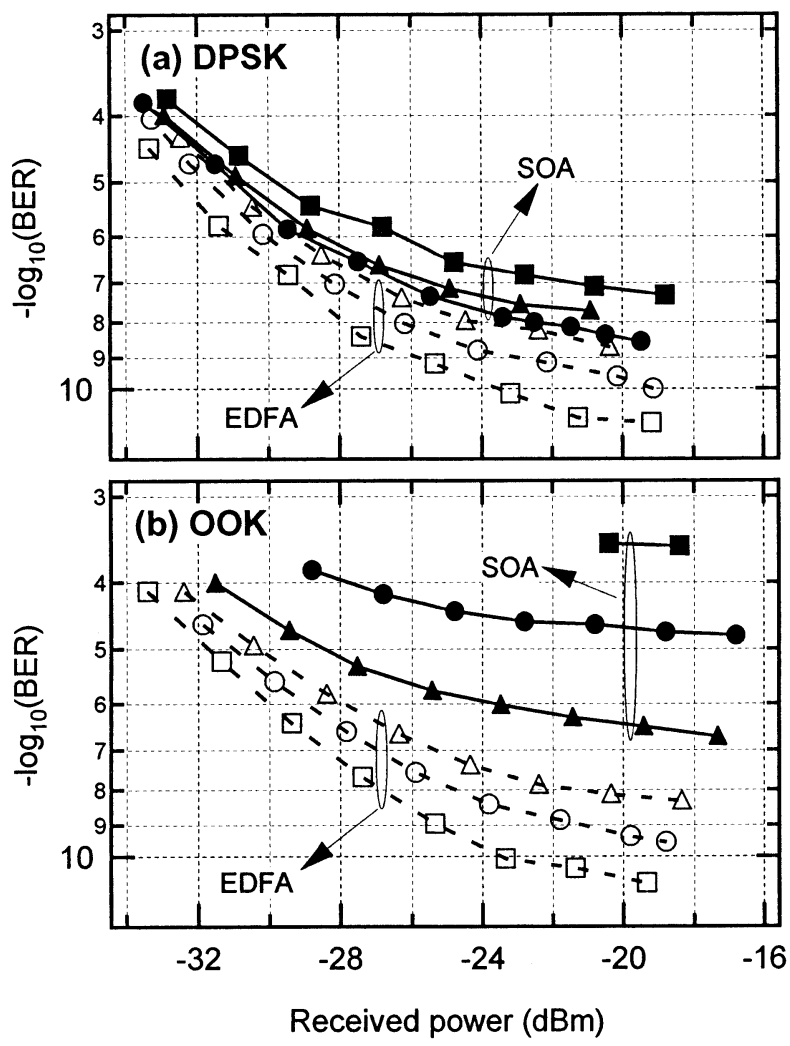

Fig. 4. (a) Measured BER of the center channel versus received power for DPSK signals through SOA or EDFA, and (b) OOK signals through SOA or EDFA. The square, circle, and triangle denote input powers of $-14,-17$, and $-20 \mathrm{dBm}$ into the SOA. The solid and dashed lines represent the cases of using SOA and EDFA, respectively. PRBS length: $2^{31}-1$.

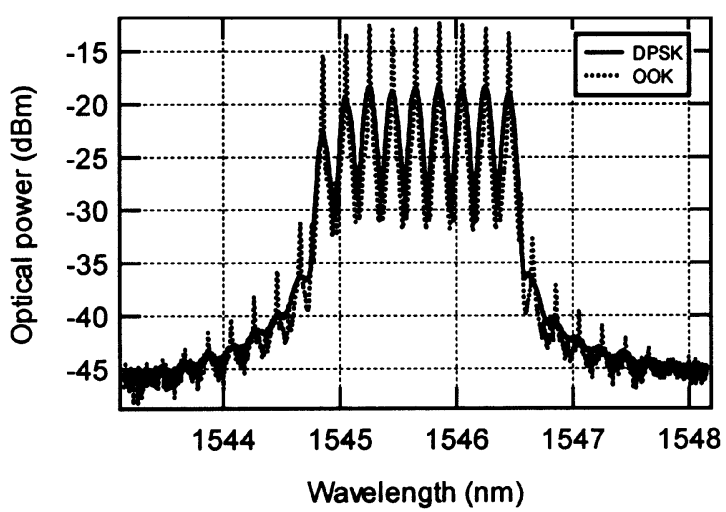

Fig. 5. Spectrum of the SOA output with nine channels of DPSK (solid) or OOK (dotted) input signals. The composite input power to the SOA was $-10 \mathrm{dBm}$ for both cases. Resolution bandwidth: $0.01 \mathrm{~nm}$.

DPSK signal, degradation of the BER was observed. This is likely caused by phase noise as a result of signal and ASE noise beating in the SOA [10]. Four-wave mixing (FWM)-induced coherent crosstalk [11] in our SOA at higher input powers, which is not negligible even with $25-\mathrm{GHz}$ channel spacing, also contributes to signal degradation as can be seen in Fig. 5. For the case of OOK, the FWM components were at least $15 \mathrm{~dB}$ below the signal channels which indicate that FWM was not a major factor to the observed pulse-pattern effect as compared with XGM.

\section{SUMMARY}

We have reported experimental results of amplification using SOA of $12.5-\mathrm{Gb} / \mathrm{s}$ DWDM signals at $25-\mathrm{GHz}$ spacing with RZ-DPSK and RZ-OOK modulation formats. Our results showed that RZ-DPSK signal is more tolerant to XGM degradation in SOA than RZ-OOK signal. However, DPSK signal suffers phase noise and FWM coherent crosstalk in SOA at high input powers similar to OOK signal. Nevertheless, DPSK modulation format should open more opportunities for applications of SOAs such as optical switching in optical cross connects and in local and metro DWDM networks.

\section{ACKNOWLEDGMENT}

The authors wish to thank Y. Achiam, M. Roberts, and C. Kerr for technical discussions and assistance.

\section{REFERENCES}

[1] M. G. Oberg and N. A. Olsson, "Crosstalk between intentisty-modulated wavelength-division multiplexed signals in a semiconductor laser amplifier," IEEE J. Quantum Electron., vol. 24, pp. 52-59, Jan. 1988.

[2] D. T. Schaafsma and E. M. Bradley, "Cross-gain modulation and frequency conversion crosstalk effects in 1550-nm gain-clamped semiconductor optical amplifiers," IEEE Photon. Technol. Lett., vol. 11, pp. 727-729, June 1999.

[3] J. B. Khurgin, I. Vurgraftman, J. R. Meyer, S. Xu, and J. U. Kang, "Reduced crosstalk semiconductor optical amplifiers based on type-II quantum wells," IEEE Photon. Technol. Lett., vol. 14, pp. 278-280, Mar. 2002.

[4] T. Durhuus, B. Mikkelsen, C. Joergensen, S. L. Danielsen, and K. E. Stubkjaer, "All-optical wavelength conversion by semiconductor optical amplifiers," J. Lightwave Technol., vol. 14, pp. 942-954, June 1996.

[5] P. S. Cho, D. Mahgerefteh, J. Goldhar, L. Joneckis, and G. Burdge, "High-performance noninterferometric semiconductor-optical-amplifier/fiber-bragg-grating wavelength converter," Electron. Lett., vol. 34, no. 4, pp. 371-373, Feb. 1998.

[6] G. Onishchuko, V. Lokhnygin, A. Shipulin, and M. Golles, "Differential binary phase-shift keying transmission using cascaded semiconductor optical amplifiers," in Proc. CLEO/Pacific Rim Conf., vol. 2, Seoul, South Korea, 1999, pp. 513-514.

[7] T. Miyano, M. Fukutoku, K. Hattori, and H. Ono, "Suppression of degradation induced by SPM/XPM+ GVD in WDM transmission using a bitsynchronous intensity modulated DPSK signal," in OECC 2000 Tech. Dig., Paper 14D3.

[8] A. H. Gnauck et al., " $2.5 \mathrm{~Tb} / \mathrm{s}(62 \times 42.7 \mathrm{~Gb} / \mathrm{s})$ transmission over $40 \times 100 \mathrm{~km}$ NZDSF using RZ-DPSK format and all-Raman-amplifed spans," in Proc. OFC 2002, Anaheim, CA, Mar. 2002.

[9] P. S. Cho, V. Grigoryan, N. Reingand, and I. Shpantzer, "Optical differential binary phase keying of return-to-zero pulses for long-haul DWDM transmission systems," presented at the 7th OptoElectronics and Communications Conf., Yokohama, Japan, July 2002, Postdeadline Paper PD-1-7.

[10] K. Kikuchi, C. Zah, and T. P. Lee, "Measurement and analysis of phase noise generated from semiconductor optical amplifiers," IEEE J. Quantum Electron., vol. 27, pp. 416-422, Mar. 1991.

[11] D. J. Blumenthal and N. C. Kothari, "Coherent crosstalk in multichannel FSK/DD lightwave systems due to four-wave mixing in semiconductor optical amplifiers," IEEE Photon. Technol. Lett., vol. 8, pp. 133-135, Jan. 1996. 